\title{
Preface (Vol 37, Issue 4)
}

\author{
Nadia Magnenat-Thalmann ${ }^{1}$
}

Accepted: 14 March 2021 / Published online: 24 March 2021

(C) The Author(s), under exclusive licence to Springer-Verlag GmbH Germany, part of Springer Nature 2021

In this issue, we first thank the reviewers for their hard work in handling papers during the year 2020 for the Visual Computer.

Then 5 papers from the special issue "Learning Representation for Visual Data" are presented. In addition, 2 papers from the conference Grapp 2019 as well as 2 papers from Cyberworlds 2019 have been selected. This issue is completed with 6 regular papers.

We also mention three corrections to 3 papers.

\section{$1 \mathrm{SI}$ : Learning representation from visual data}

We are happy to present 5 papers for the special issue "Learning representation from visual data" that has been handled by 4 guest editors who are:

- Dr. Wenming Zheng, Southeast University, China (lead Editor and Associate Editor of the Visual Computer).

- Dr. Jianjun Qian, Nanjing University of Science and Technology (guest editor).

- Dr. Wankou Yang, Southeast University, China (guest editor).

- Dr. Richard Yi Da Xu, University of Technology, Sydney (guest editor).

After a very careful review, the following papers have been selected:

1. Qiao Du et al., titled: "Block dictionary learning-driven convolutional neural networks for few shot face recognition".

Nadia Magnenat-Thalmann

thalmann@miralab.ch

1 MIRALab-CUI, University of Geneva, Battelle, Building A,

7, Route de Drize, 1227 Carouge, Geneva, Switzerland
2. Li Zhang et al., titled: "Dissimilarity-based nearest neighbor classifier for single-sample face recognition".

3. Cairong Zhao et al., titled: "FLAG: feature learning with additional guidance for person search".

4. Qi Zhu et al., titled: "An effective recognition approach for contactless palm print".

5. Azadeh Montazeri et al., titled: "MLK-SVD, the new approach in deep dictionary learning".

\section{Special selection from VISIGRAPP 2019 Conference}

We are happy to present 2 selected papers from the conference VISIGRAPP 2019, chaired by Jose Braz from Portugal

1. Felix Gaisbauer et al., titled: "Combining heterogeneous digital human simulations: presenting a novel cosimulation approach for incorporating different character animation technologies".

2. Bedrich Benes et al., titled: "Character motion in function space".

\section{Special selection from CYBERWORLDS 2019 Conference}

We are happy to present 2 selected papers from the CYBERWORLDS 2019 Conference, chaired by Alexei Sourin, NTU, Singapore

1. Xingce Wang et al., titled: "3D skull and face similarity measurements based on a harmonic wave kernel signature".

2. Xiaoyang Mao et al., titled: "Matching a composite sketch to a photographed face using fused HOG and deep feature models". 


\section{Regular papers}

The following 6 papers have been submitted as regular papers to the Visual Computer

1. Naoki Kita et al., titled: "Computational design of polyomino puzzles" (also presented at CGI2020 conference).

2. Mehdi Ayadi et al., titled: "A skyline-based approach for mobile augmented reality".

3. Hongbo Huang et al., titled: "YOLO-face: a real-time face detector".

4. Jinlong Shi et al., titled: "A self-supervised method of single-image depth estimation by feeding forward information using max-pooling layers".

5. Nam Kim et al., titled: "Efficient object tracking using hierarchical convolutional features model and correlation filters".

6. Selçuk Aslan et al., titled: "Modified artificial bee colony algorithms for solving multiple circle detection problem".

\section{Corrections}

We mention three corrections to the following papers:

1. Kwnag Ko et al., Correction to: Automated recognition of 3D pipelines from point clouds.

2. Jianping Hu et al., Correction to: Robust and blind image watermarking via circular embedding and bidimensional empirical mode decomposition.

3. Yan Jin et al., Correction to: Low-dose CT lung images denoising based on multiscale parallel convolution neural network.

Publisher's Note Springer Nature remains neutral with regard to jurisdictional claims in published maps and institutional affiliations. 